ders./V. Brandes (Hrsg.), Handbuch 2 Unterentwicklung, Frankfurt/M. 1975, S. 64-86). Diese Kritik wird von Ibrahim und Metze-Mangold als sachlich nicht gerechtfertigt und in der Darstellung verfälschend zurückgewiesen (S. 115 ff.). Die Verf. versuchen demgegenüber dem NKEW sachlich gerecht zu werden, indem sie auf die ideengeschichtlichen Ursprünge dieser Theorie eingehen (Kap. 1). In einem weiteren Kapitel werden deren sozioökonomische und politische Elemente skizziert. Die Verf. verzichten dabei allerdings nicht auf Kritik. Insbesondere wird der NKEW-Theorie vorgehalten, die realen politisch-ökonomischen Entwicklungen in Entwicklungsländern, die einen solchen Entwicklungsweg zeitweilig eingeschlagen hatten (etwa Ägypten), analytisch nicht zufriedenstellend zu beschreiben (S. $117 \mathrm{ff}$.). Da das vorliegende Buch eine vorhandene wesentliche Lücke schließt, ist es als Einführung in die Theorie des NKEW sehr zu empfehlen.

Alphons Studier

RiCHARd A. JOSEPH

\title{
Radical Nationalism in Cameroun
}

Social Origins of the UPC Rebellion

Oxford: University Press 1977, 383 S., $15 £$

Bei dieser Arbeit handelt es sich um einen empirischen Beitrag zum Problem des anti-kolonialen afrikanischen Nationalismus, zum Dekolonisierungsprozeß sowie zum Problem des Neo-Kolonialismus, d. h. der kolonialen Festlegung nach-kolonialer, ,, neo-kolonialer“ politischer Strukturen. Gegenstand der Untersuchung ist die radikal anti-koloniale, aber nicht unbedingt ,,kommunistische“ (wie der Autor überzeugend darlegt), ,Union des Populations du Cameroun“ (UPC) (seit 1948). Deren historische Basis sowie spätere Entfaltung bis zu deren aktivem Beginn einer Busch-Guerilla (Dezember 1956) werden hier erstmals in einer überzeugenden, wohl abgewogenen, alle verfügbaren Quellen sowie zahlreiche persönliche Interviews mit Beteiligten in Paris und Kamerun auswertenden Weise in ihrer ökonomischen, sozialen und politischen Dimension dargestellt. Der Autor kommt dabei u. a. zu einer Neubewertung der Rolle der Jeunesse Camerounaise Française (1938 ff.) sowie der Rolle der französischen Colons - auch in ihrem Spannungsverhältnis zu den jeweiligen Administrationen - im Lande. Er zeigt, wie die radikal-antikoloniale UPC den Seitenwechsel der von Houphouet-Boigny geführten RDA nicht mitmachte, wie sie, trotz vielfältiger französischer Repressionsmaßnahmen, ihre Basis immer weiter auszubauen vermochte. Erst durch die Provozierung von Zwischenfällen und Illegalisierung sowie schließlich durch die Aufreibung in einem langjährigen Buschkrieg (auf den der Verfasser nur in einem kurzen Epilog eingeht) vermochte die Kolonialmacht diese Partei letztlich erfolgreich aus dem politischen Leben auszuschalten und den mit ihr kooperationsbereiten konservativen Kräften, erst um Mbida, dann um Ahidjo, einen Teil der Macht und schließlich die politische Nachfolge (in die völkerrechtliche Unabhängigkeit) zu übergeben. Es ist vielleicht nicht ohne Pikanterie - worauf die auch in dieser Zeitschrift schon besprochene Arbeit von Beuth hingewiesen hat (vgl. VRU 1977, S. 161) - daß der Mann der Franzosen - Ahidjo-dann allerdings nur so lange den Erwartungen seiner Mentoren voll entsprach, wie er diese zur Unterdrükkung und endgültigen Ausschaltung der UPC tatsächlich brauchte. Als dieses Problem (militärisch) endgültig gelöst war, war auch er gewillt, eine in vielen politischen Fragen recht unabhängige Haltung einzunehmen, unabhängiger jedenfalls, als viele seiner Kollegen in den anderen frankophonen Ländern.

Rolf Hanisch 